\title{
Combined pharmacological and motor training interventions for recovery of upper limb function in subacute ischemic stroke
}

\author{
Ioana Stanescu ${ }^{1,4}$, Gabriela Dogaru ${ }^{2,4}$, Adriana Bulboaca ${ }^{3,4}$, Adina Stan ${ }^{1,5}$, Delia Stanca ${ }^{1,5}$, Alina Blesneag ${ }^{1,5}$, \\ Rita Kallo ${ }^{4}$ \\ 1. Depart. of Clinical Neurosciences, “Iuliu Hatieganu” University of Medicine and Pharmacy, Cluj Napoca \\ 2. Depart. of Physical Med. and Rehab., "Iuliu Hatieganu” University of Medicine and Pharmacy, Cluj \\ Napoca \\ 3. Dep. of Physiopathology, "Iuliu Hatieganu” University of Medicine and Pharmacy, Cluj Napoca \\ 4. Rehabilitation Hospital Cluj Napoca \\ 5. Neurology Clinic, County Emergency Clinical Hospital Cluj Napoca
}

\begin{abstract}
Motor deficit, especially in the upper limb, is the primary contributor in post-stroke disability. Recovery of motor function relies on neural plasticity - cortical plastic reorganization - a spontaneous process, which could be enhanced from early phases by rehabilitative strategies. The subacute stage after stroke is the critical period during which the brain is most receptive to rehabilitation strategies. Based on the recent results of 2 trials in stroke rehabilitation using pharmacological intervention with Cerebrolysin in combination with standardized kinesitherapy, we conducted a pilot study of 4 consecutive patients with acute ischemic stroke, treated with Cerebrolysin for 28 days after stroke, and with intensive taskspecific kinesitherapy from day 7 to day 28 after stroke. We assessed stroke severity with NIHSS score, upper limb function with ARAT (Action Research Arm Test) score, disability with modified Rankin scale and patient's autonomy with Barthel Index, at day 0 and day 30 after stroke. After 28 days of combined therapy all 4 patients improved, most significant improvement was seen in upper limb function, measured by ARAT score and in autonomy measured by Barthel Index.
\end{abstract}

Keywords: stroke rehabilitation, neural plasticity, Cerebrolysin, motor recovery

\section{INTRODUCTION}

Motor deficit, especially in the upper limb, is the primary contributor in post-stroke disability.

Recovery of strength and motor function is mainly attributed to cortical plastic reorganization in the early recovery phase. Neural plasticity is the ability of brain to develop new neuronal interconnections, acquire new functions, and compensate for impairments [1], which will change the structure and function of cortical neurons and networks. After focal damage of the motor cortex, structural and functional reorganization occurs ipsilateral in perilesional suriving areas, but also in the contralateral hemisphere. Neural plasticity is a spontaneous process, based on innate plasticity, which could be increased via rehabilitative interventions, such as pharmacological agents, task-specific motor training or non-invasive brain stimulation.

The practical goal of cortical plastic reorganization after motor cortex injury is recovery of functional motility of hemiplegic/paretic side (motor rehabilitation). This goal is achieved by (a) spontaneous recovery and (b) motor learning.

Motor rehabilitation relies on a combination of different processess: recovery, substitution and compensation [2]. Motor recovery means that undamaged brain regions are still able to command same muscle groups as before the injury to produce the same motor patterns. Motor compensation refers to activation of alternative brain areas which will stimulate different muscle groups in order to produce new motor patterns for the same task.

a). Spontaneous biological recovery begins immediately after stroke, is maximal during the first 3-4 weeks, continue with high 
intensity in the first 3 months post-stroke and tapers progressively over 6 months. The subacute stage after stroke is the critical period during which the brain is most receptive to changements by rehabilitation strategies. However, the recovery process did not end at that moment, studies have shown that repetitive, high intensity, task-specific motor practice could improve motor function even in chronic stages (after 1 year) of stroke. [3]

b). Motor learning process is based on structural neuronal changes, as dendrite sprouting, formation of new synapses or neurochemical changes [4].

Motor learning have better results if the methods used are meaningful for the patient, are performed repetitively and intensively in an enriched environment [4].

The essential strategies used for motor rehabilitation after stroke are task-specific motor training and an enriched environment. Task-specific training means that the motor training should be oriented to achieve the goals that are relevant to the functional needs of the patient. Compared with traditional simple motor exercises, task-specific training induces long-lasting motor learning and adaptative cortical reorganization by neural plasticity [5], [6]. Enriched environment should provide many opportunities for physical activity and for motivation [7].

The techniques used in motor learning-based rehabilitation strategies are: constraintinduced movement therapy (CIMT), body weight-supported treadmill training (BWSTT), robotic training, noninvasive brain stimulation (NIBS), transcutaneous neuromuscular electrical stimulation. All of these methods have evidence showing increasing in neural plasticity process [4].

Classical physical therapy techniques or simple motor exercises combined with occupational therapy are still used, but their effect is shorter and limited. Stroke survivors with reduced strength in their arms or legs should be offered progressive resistance training [8], [9]. In people with mild to severe arm weakness after stroke, mechanically assisted arm training (e.g. robotics) should be used to improve upper limb function [10].

Multisensory feedback plays also a role in motor learning by reestablishing the disrupted sensorimotor loop after stroke. Multisensory feedback - based rehabilitation strategies are: action observation, mental practice, mirror therapy, virtual reality (VR) training, and brain-computer interface (BCI) [4], [11].

Duration of the rehabilitation therapy is not clearly established: for stroke survivors, rehabilitation should provide as much scheduled therapy (occupational therapy and physiotherapy) as possible, with a minimum of three hours a day [12].

Pharmacological interventions improves motor learning in stroke patients. Clinical trials suggest that pharmacological interventions may facilitate physical therapy and enhance the effect of rehabilitative techniques for motor learning. Pharmacological agents are the key factors for succesfull intervention in rehabilitation by modulation and support for the endogenous defense activities that are induced by damage mechanisms after stroke.

Cerebrolysin is a neuropeptide preparation which consists is low molecular weight neuropeptides and free amino acids; its multimodal compounds have effect on many associated pathophysiologic events. Cerebrolysin have neuroprotective properties, neurotrophic activity, promote neuronal sprouting, improve cellular surival and stimulate neurogenesis, being a multitargeted therapy by its multimodal compounds [13]. Recently published CARS trial shows that Cerebrolysin treatment associated with intensive kinesitherapy has beneficial effects on upper limb motor function (as measured by ARAT score) and on global outcome in early rehabilitation period after stroke [13]. The ECOMPASS trial published on 2016 [14] shows that adding Cerebrolysin treatment on the top of rehabilitation therapy program has a beneficial effect on motor recovery in patients with severe motor impairment in subacute phase of ischemic stroke. The optimization of motor recovery was attributed by 
neuroimagistic techniques to the enhancement of motor network plasticity [14].

Despite the progresses made in the field of post-stroke motor rehabilitation, patients' response to rehabilitative interventions is highly variable, depending on stroke severity (lesion size and location, corticospinal damage/integrity), injuries of other brain structures, age and psychosocial factors.

\section{CASE PRESENTATION:}

Starting from the hypothesis of synergistic effect of a combination of rehabilitation methods in improving motor deficit after stroke, as demonstrated recently in CARS and ECOMPASS studies, we used pharmacological intervention and standardized rehabilitation program in a group of 4 patients in acute and subacute phase of ischemic stroke.

We selected 4 consecutive patients with first acute ischemic stroke, cortical or subcortical localization (in the carotid artery territory), confirmed by CT scan, without significant pre-stroke disability. Patients were included if they arrived in the first 24 hours after stroke, and were no candidates for thrombolytic treatment.

Severity of stroke was assessed using the NIHSS score.

Upper limb motor function was assessed using the ARAT - Action Research Arm Test score [15] - with a maximum score of 57 points (normal upper limb function) and a minimum of 0 points (complete plegia).

Disability was assessed with modifyed Rankin scale (mRS), and patient's autonomy with Barthel Index (BI).
All assessments were made at day 1 and day 30 after stroke.

For pharmacological intervention we used Cerebrolysin, dose of $30 \mathrm{ml} /$ day diluted with physiological saline to a total volume of 100 $\mathrm{ml}$-in intravenous administration, for a period of 28 days, starting within 24 hours after stroke onset.

Rehabilitative interventions were started in the 7-th day after stroke onset, when the patient was transferred in Rehabilitation Hospital; consist in standardized physiotherapy program for 2 hours/day (using task-specific motor training) and occupational therapy for 1 hour/day, 5 days/week, for 3 weeks.

Informed consent was obtained for all 4 patients participating in the study. The pilot study has receved the approval of the Local Ethic Comission.

Case 1: Patient D.I., male, 61 years, diagnosed with ischemic stroke in the left middle cerebral artery (MCA) territory. Clinical evaluation at day 1 shows right hemiparesis with brachial predominance (force MRC $=3 / 5$ upper limb, 4/5 lower limb) and moderate expressive aphasia. Risk factors were treated hypertension, diabetes, and dyslipidemia.

Paraclinical investigations reveal a stenosis of left internal carotid artery (ICA) of $70 \%$, a right ICA stenosis of $50 \%$ and a supraventricular arrythmia, without atrial fibrillation (AF) at Holter ECG. Stroke etiology is presumed to be aterothrombotic. CT scan on Day 0 is shown in Figure 1:

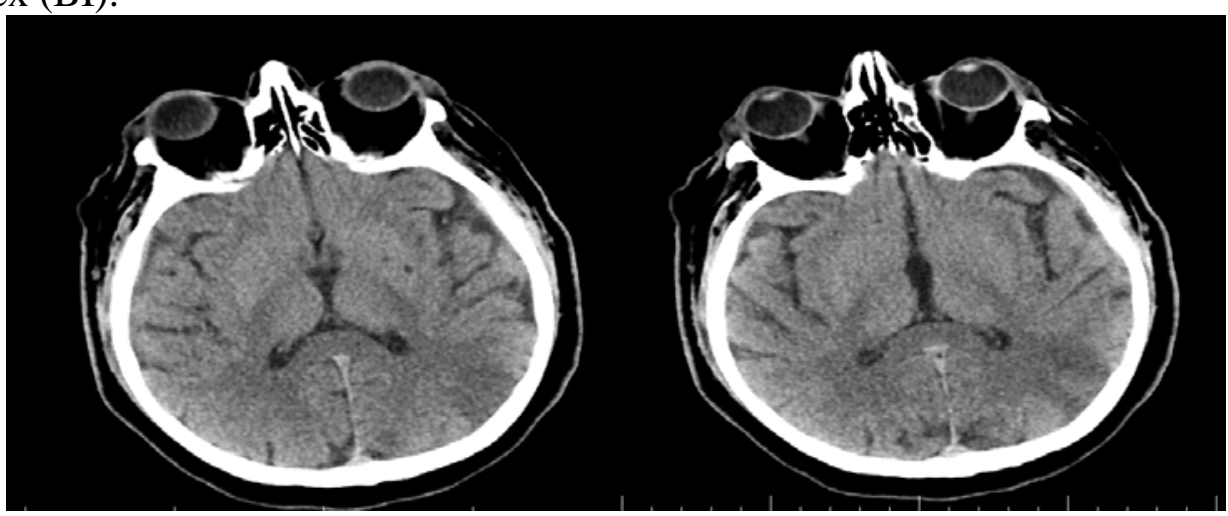

Figure 1: CT scan, day 0, patient nr. 1 
At day 30, neurological status of the patient improved to a mild hemiparesis (force MRC = 4/5 upper limb, 5/5 lower limb) - NIHSS score $=5$ points. Upper limb function also improved, as shown by the increase of ARAT score to 47 points, as shown in table 1 .

\begin{tabular}{|c|c|}
\hline ASSESSMENT D1 CASE 1 & ASSESSMENT D 30 CASE 1 \\
\hline NIHSS $=8 \mathrm{pts}$ & NIHSS $=5 \mathrm{pts}$ \\
\hline ARAT score $=\mathbf{3 8}$ & ARAT score $=\mathbf{4 7}$ \\
\hline $\mathrm{mRS}=4$ & $\mathrm{mRS}=3$ \\
\hline Barthel index $=45 \mathrm{pts}$ & Barthel index $=70 \mathrm{pts}$ \\
\hline
\end{tabular}

Table 1: Parameters assessed at day 0 and day 30 for patient nr.1

Case 2: Patient O.N., male, 68 years, diagnosed with ischemic stroke in the superficial right MCA territory. Neurological examination shows severe left hemiparesis (MRC 2/5 upper limb and 3/5 lower limb). Risk factors were hypertension and smoking. Paraclinical investigations did not point to a specific etiology.

CT scan on Day 0 is shown in Figure 2:
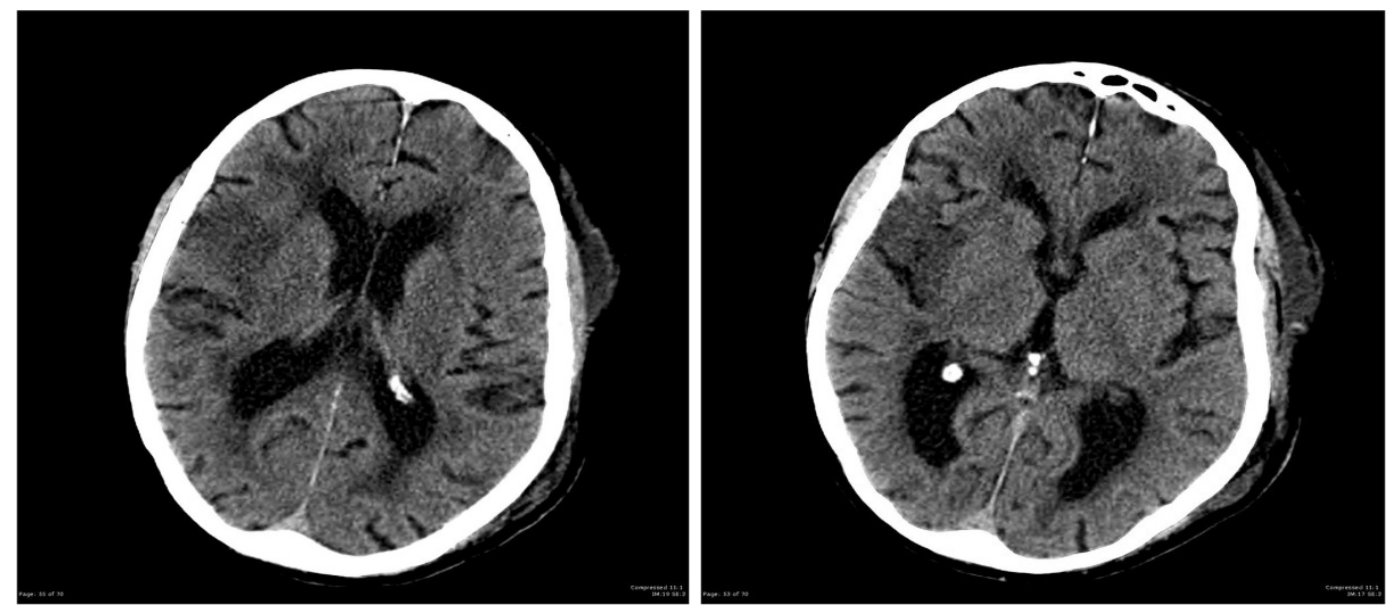

Figure 2: CT scan, day 0, patient nr. 2

At day 30, after combined pharmacological and task-specific training interventions, neurological status of the patient shows a moderate improvement in the NIHSS score (to a force MRC=3/5 in upper limb, and 3/5 in lower limb), and in the ARAT score which increased from 2 pts to 4 points. Patient's autonomy was also slightly improved. All data are shown in table 2.

\begin{tabular}{|c|c|}
\hline ASSESSMENT D1 CASE 2 & ASSESSMENT D 30 CASE 2 \\
\hline NIHSS $=10 \mathrm{pts}$ & NIHSS $=6$ pts \\
\hline ARAT score $=\mathbf{2}$ & ARAT score $=\mathbf{4}$ \\
\hline $\mathrm{mRS}=5$ & mRS $=4$ \\
\hline Barthel index $=15 \mathrm{pts}$ & Barthel index $=30 \mathrm{pts}$ \\
\hline
\end{tabular}

Table 2: Parameters assessed at day 0 and day 30 for patient nr.2 
Case 3: Patient M.L., 58 years, diagnosed with ischemic stroke in the right MCA territory. Neurological examination shows moderate left hemiparesis ( force MRC $=3 / 5$ upper limb, 4/5 lower limb), left homonimous hemianopia, neglect on the left side of his body. Risk factors were recent
$(<24 \mathrm{~h})$ miocardial infarctus treated with PTCA + stent and controlled arterial hypertension. Stroke etiology was considered embolic.

CT scan day 0 is shown in figure 3:

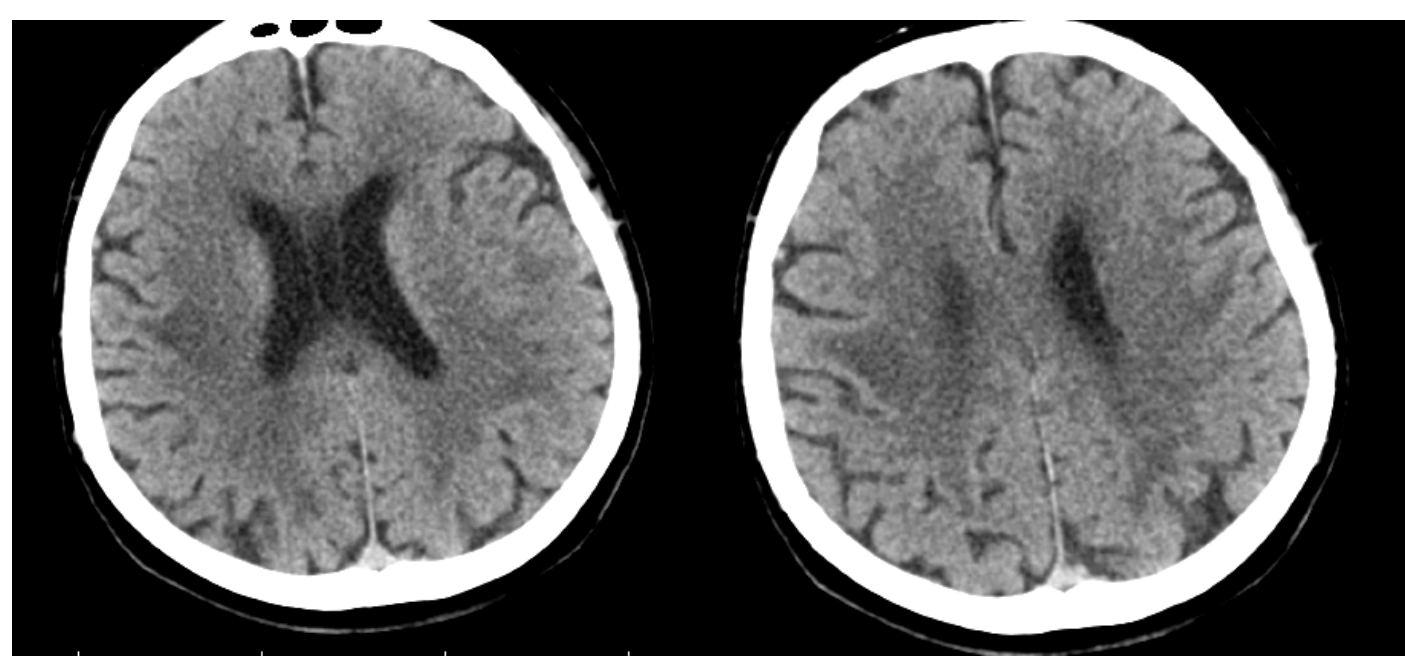

Figure 3: CT scan, day 0, patient nr. 3

At day 30, after rehabilitation program, neurological status of patient markedly improved. Neurological examination showed mild left quadrantanopia, mild left hemiparesis (MRC $=4+/ 5$ upper limb and $5 / 5$ lower limb) and no neglect. A spactaculr improvement was seen on upper limb function, which recovers from 9 points on ARAT scale to 53 points. Patient's disability drecreases to 1 point on modufyed Rankin scale. Patient regained almost complete autonomy, as shown by improvement in Barthel index. Parameters are shown in table 3.

\begin{tabular}{|c|c|}
\hline ASSESSMENT D1 CASE 3 & ASSESSMENT D 30 CASE 3 \\
\hline NIHSS $=7$ pts & NIHSS = 3 pts \\
\hline ARAT score $=\mathbf{9}$ & ARAT score $=\mathbf{5 3}$ \\
\hline mRS $=\mathbf{4}$ & mRS $=\mathbf{1}$ \\
\hline Barthel index $=\mathbf{3 0}$ pts & Barthel index $=\mathbf{7 5}$ pts \\
\hline
\end{tabular}

\section{Table 3: Parameters assessed at day 0 and day 30 for patient nr.3}

Case 4: M.I., 74 years, diagnosed with ischemic stroke in left posterior cerebral artery (PCA) territory. Neurologic examination at admission showed right severe hemiparesis with brachial predominance (force MRC = 2/5 upper limb, 3/5 lower limb), Wernicke aphasia, right homonymous hemianopia. Risk factors were uncontrolled hypertension, smoking and chronic alcohol comsumption. Paraclinical investigations found moderate stenosis on both internal carotid arteries, stroke etiology is presumed to be aterothrombotic.

Patient CT scan at admission is shown in figure 4: 


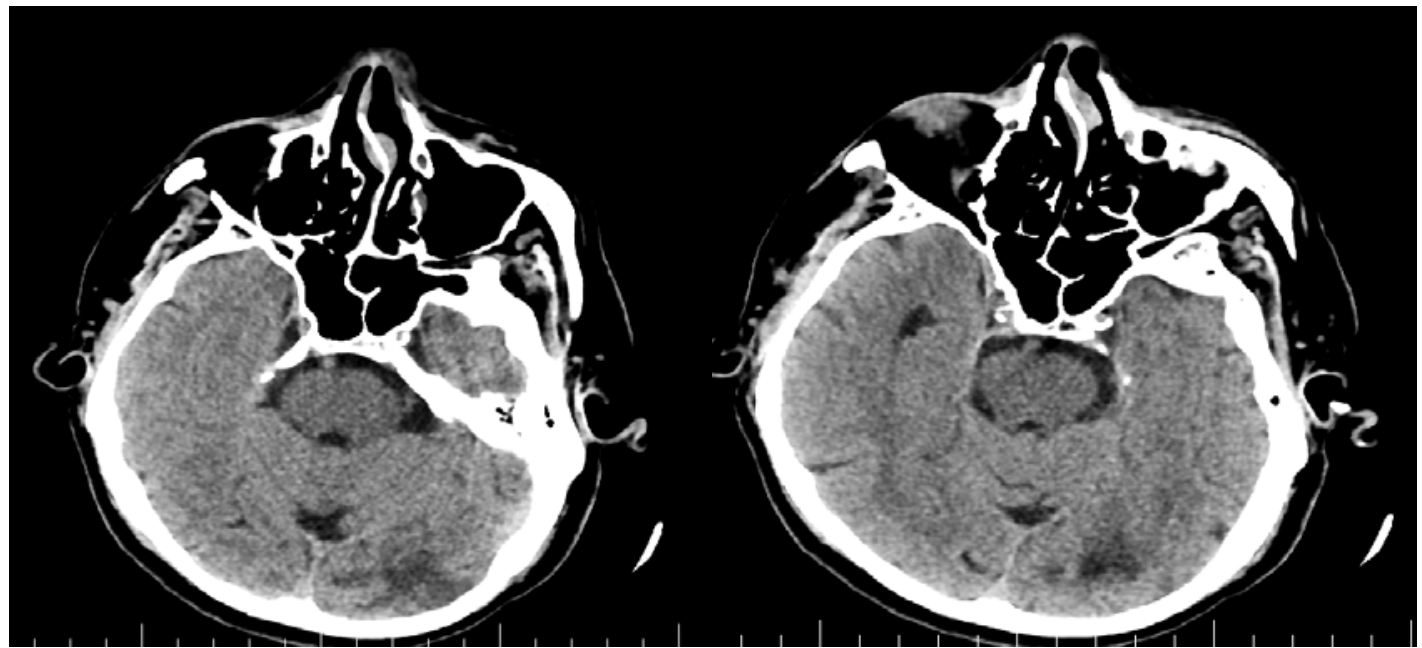

Figure 4: CT scan, day 0, patient nr. 4

At day 30, neurological status of this patient also improved, showing a mild right hemiparesis (MRC force right upper and lower limb $=4 / 5)$, moderate Wernicke aphasia, right homonymous hemianopia.Important improvement was seen on upper limb function, as measured by ARAT scale, which improved from 0 points, meaning plegia, to 36 points. Disability improves from severe to moderate, and autonomy improves from 5 points at Barthel Index to 40 points (data shiwn in table 4).

\begin{tabular}{|c|c|}
\hline ASSESSMENT D1 CASE 4 & ASSESSMENT D 30 CASE 4 \\
\hline NIHSS $=17 \mathrm{pts}$ & NIHSS $=6$ pts \\
\hline ARAT score $=\mathbf{0}$ & ARAT score $=\mathbf{3 6}$ \\
\hline mRS $\mathbf{5}$ & mRS $=\mathbf{3}$ \\
\hline Barthel index $=\mathbf{5}$ pts & Barthel index $=\mathbf{4 0}$ pts \\
\hline
\end{tabular}

\section{Table 4: Parameters assessed at day 0 and day 30 for patient nr.4}

\section{CONCLUSION:}

In conclusion to this observational study, all 4 patients improved after 28 days of

combined pharmacotherapy with Cerebrolysin $30 \mathrm{ml} / \mathrm{d}$ and 21 days of intensive physical and ocupational therapy (3h/day) in a Rehabilitation facility. .

Among the studied parameters, the most significant improvement was seen in upper limb function, measured by ARAT score and in autonomy measured by Barthel Index.

The strength of our approach in rehabilitation of patients with acute ischemic stroke was the combination between high doses of Cerebrolysin administered for 28 days in the first 24 hours after stroke and high intensity task-specific kinesi- and occupational therapy, administered for 21 days in a Rehabilitation Hospital.
The limitations of our observational study were the small number of patients, the selection of cases, the absence of a control group, different location of strokes and the presence of other neurological deficits than motor deficit that influenced the degree of disability and autonomy.

We hope that the advances in stroke rehabilitation strategies will show new evidence about the efficacy of combination therapies on facilitation and modulation of neural plasticity, with improvement of motor function, beginning from the first days after stroke onset. 


\section{BIBLIOGRAPHY}

1. Murphy TH, Corbett D. Plasticity during stroke recovery: from synapse to behavior. Nat Rev Neurosci. 2009 Dec;10(12):861-72.

2. Li S. Spasticity, Motor Recovery, and Neural Plasticity after Stroke. Front.Neurol. 2017; 8:120

3. Page SJ, Gater DR, Bach YRP. Reconsidering the motor recovery plateau in 11 .

stroke rehabilitation. Arch Phys Med Rehabil. Takeuchi N, Izumi S-I. Combinations of 2004; 85:1377-81

4. Takeuchi N, Izumi SI. Rehabilitation with Poststroke Motor Recovery: A Review with a Focus on Neural Plasticity. Stroke Res Treat. 2013:12864

5. Richards LG, Stewart KC, Woodbury ML, Senesac C, Cauraugh JH. Movementdependent stroke recovery: a systematic review and meta-analysis of TMS and fMRI evidence. Neuropsychologia. 2008 Jan 15;46(1):3-11.

6. Bayona NA, Bitensky J, Salter K, Teasell $\mathrm{R}$. The role of task-specific training in rehabilitation therapies. Top Stroke Rehabil. 2005 Summer;12(3):58-65. Review. 7. Döbrössy MD, Dunnett SB. The influence of environment and experience on neural grafts. Nat

$\operatorname{Rev}$

Neurosci. 2001 Dec;2(12):871-9

8. Ada L, Dorsch S, Canning CG. Strengthening interventions increase strength and improve activity after stroke: a systematic review. Aust

Physiother.2006;52(4):241-8

10. $\mathrm{Me}$

hrholz J , Pohl M, Platz T, Kugler J, Elsner B. Electromechanical and robot-assisted arm training for improving activities of daily living, arm function, and arm muscle strength after stroke. Cochrane Database Syst Rev. 2015 Nov 7;(11) :CD006876 stroke neurorehabilitation to facilitate motor recovery: perspectives on Hebbian plasticity and homeostatic metaplasticity. Front. Hum. Neurosci. 9:349

12.Lohse $\mathrm{KR}$, Lang CE, Boyd LA. Is more better? Using metadata to explore doseresponse relationships in stroke rehabilitation. Stroke. 2014 Jul;45(7):2053-8 13.Muresanu DF, Heiss WD, Hoemberg V, Bajenaru O, Popescu CD, Vester JC, Rahlfs VW, Doppler E, Meier

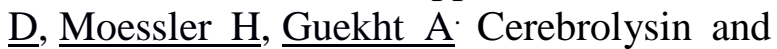
Recovery After Stroke (CARS): A Randomized, Placebo-Controlled, DoubleBlind, Multicenter Trial. Stroke. 2016 Jan;47(1):151-9

14.

ng WH, Park CH, Kim DY, Shin YI, Ko $\underline{\mathrm{MH}}$, Lee A, Jang SY, Kim YH. Cerebrolysin combined with rehabilitation promotes motor recovery in patients with severe motor impairment after stroke. $\underline{B M C}$ Neurol. 2016 Mar 2;16:31

15.

9. Harris JE, Eng JJ. Strength training improves upper-limb function in individuals with stroke: a meta-analysis. Stroke. 2010 Jan;41(1):136-40

e RC. A performance test for assessment of upper limb function in physical rehabilitation treatment and research. Int $J$ Rehabil Res. 1981;4:483-49 